\title{
Determination of low-energy constants of Wilson chiral perturbation theory
}

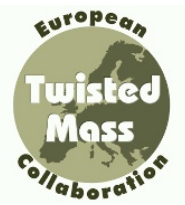

\section{The ETM collaboration}

\section{Gregorio Herdoíza, ${ }^{a, b, 1}$ Karl Jansen, ${ }^{c, d}$ Chris Michael, ${ }^{e}$ Konstantin Ottnad ${ }^{f}$ and} Carsten Urbach ${ }^{f}$

${ }^{a}$ PRISMA Cluster of Excellence, Institut für Kernphysik, Johannes Gutenberg-Universität, D-55099 Mainz, Germany

${ }^{b}$ Departamento de Física Teórica and Instituto de Física Teórica UAM/CSIC, Universidad Autónoma de Madrid, Cantoblanco, E-28049 Madrid, Spain

${ }^{c}$ NIC, DESY,

Platanenallee 6, D-15738 Zeuthen, Germany

${ }^{d}$ Department of Physics, University of Cyprus, P.O.Box 20537, 1678 Nicosia, Cyprus

e Theoretical Physics Division, Department of Mathematical Sciences, The University of Liverpool, Liverpool L69 3BX, U.K.

${ }^{f}$ Helmholtz-Institut für Strahlen- und Kernphysik (Theorie) and Bethe Center for Theoretical Physics, Universität Bonn, 53115 Bonn, Germany

E-mail: herdoiza@kph.uni-mainz.de, karl.jansen@desy.de,

c.michael@liv.ac.uk, ottnad@hiskp.uni-bonn.de,

urbach@hiskp.uni-bonn.de

ABSTRACT: By matching Wilson twisted mass lattice QCD determinations of pseudoscalar meson masses to Wilson Chiral Perturbation Theory we determine the low-energy constants $W_{6}^{\prime}, W_{8}^{\prime}$ and their linear combination $c_{2}$. We explore the dependence of these low-energy constants on the choice of the lattice action and on the number of dynamical flavours.

KEYWORDS: Lattice QCD, Chiral Lagrangians

ARXiv EPRINT: 1303.3516

\footnotetext{
${ }^{1}$ Corresponding author.
} 


\section{Contents}

1 Introduction 1

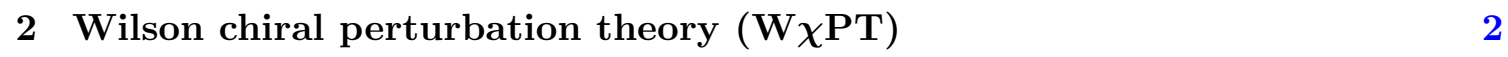

3 Lattice actions $\quad 4$

3.1 Wilson twisted mass fermions 4

$\begin{array}{lll}3.2 & \text { Gauge action } & 6\end{array}$

4 Numerical studies $\quad 7$

4.1 $N_{\mathrm{f}}=2+1+1 \mathrm{Wtm}$ fermions with Iwasaki gauge action $\quad 7$

$4.2 N_{\mathrm{f}}=2 \mathrm{Wtm}$ fermions with tlSym gauge action 11

5 Discussion $\quad 14$

$\begin{array}{llr}6 & \text { Conclusions } & 16\end{array}$

\section{Introduction}

Lattice QCD simulations employing a discretisation of the Dirac operator based on the original proposal by Wilson [1] are currently being performed with light dynamical fermions [2-9]. When decreasing the light quark mass at a fixed value of the lattice spacing, a subtle interplay between mass and discretisation effects can take place due to the explicit breaking of chiral symmetry by the Wilson term. In simulations with light values of the quark mass it is, therefore, vital to understand and monitor the discretisation effects and to obtain a quantitative measure of their size.

Close to the continuum limit, a useful way to determine the discretisation effects in the regime of light quark masses is provided by Wilson chiral perturbation theory (W $\chi \mathrm{PT}$ ), an extension of the continuum chiral effective theory including additional terms proportional to powers of the lattice spacing [10]. Depending on the order of the expansion, additional low energy constants (LECs) appear, whose values are not known a priori: they depend on the lattice action and can only be computed from a simulation.

Knowing the values of the LECs of $\mathrm{W} \chi \mathrm{PT}$ is of particular interest, because $\mathrm{W} \chi \mathrm{PT}$ predicts a non-trivial phase structure for Wilson type fermions in the lattice spacing and quark mass plane [10-12]. Depending on the sign of a particular combination of Wilson LECs - commonly denoted as $c_{2} \propto-\left(2 W_{6}^{\prime}+W_{8}^{\prime}\right)$ - either the Aoki-scenario [11] or a first order, so called Sharpe-Singleton [10] scenario is realised. Numerical evidence for both scenarios has been observed in lattice QCD simulations and dedicated studies of the associated phase diagrams have been performed by several groups [13-24]. 
The Aoki scenario with a positive $c_{2}$ was found to be realised in quenched simulations. In dynamical simulations the Sharpe-Singleton scenario with negative $c_{2}$ was observed when using Wilson twisted mass fermions at maximal twist [20-23]. This manifests itself in the fact that the neutral pion mass $M_{\pi^{0}}$ is lighter than the charged one, $M_{\pi^{ \pm}}$, where the splitting of the squared masses is proportional to $c_{2}$. In turn, a measurement of the pion mass-splitting in Wilson twisted mass lattice QCD provides a way to measure $c_{2}$ and, hence, the LECs of the corresponding chiral effective theory. However, this way of computing $c_{2}$ is challenging since for the neutral pion mass disconnected contributions need to be evaluated.

There are also alternative ways to determine the LECs of W $\chi \mathrm{PT}$. In refs. [25-28] they have been studied by matching the analytical predictions [27, 29-36] for the spectrum of the Wilson Dirac operator - with fixed index in a finite volume - to lattice data. ${ }^{1}$ Determinations of the Wilson LECs have also been carried out via the spectral density of the Hermitian Wilson-Dirac operator [38-40]. Lattice determinations of the pion scattering lengths have been used in refs. [41, 42], an approach that was extended to a partially quenched setup in ref. [43]. In a mixed action with Wilson-type sea fermions and chirally invariant valence quarks, a mixed action chiral Lagrangian [44, 45] can be constructed. The corresponding LECs - in particular $W_{8}^{\prime}$ — have been recently determined in the case of overlap valence quarks on a Wilson twisted mass sea [46].

In this paper we are going to determine the LECs using a method recently proposed in ref. [47]. It relies on the measurement of pseudoscalar meson masses involving Wilson twisted mass fermions. In this approach, the LEC $W_{8}^{\prime}$ is related to the mass-splitting between the charged pion mass $M_{\pi^{ \pm}}$and the "connected neutral pion" mass $M_{\pi^{(0, \mathrm{c})}}$. The latter is determined from the quark-connected correlation, which contributes to the neutral pion in twisted mass QCD. The "connected neutral pion" correlation function thus differs from the complete correlation function needed to determine the neutral pion by the absence of disconnected diagrams. This has the advantage that in numerical studies the "connected pion mass-splitting" should be accessible with good statistical precision. In addition, the mass-splitting, $M_{\pi^{(0, c)}}^{2}-M_{\pi^{0}}^{2}$, between the connected and full neutral pion mass, provides an estimate of $W_{6}^{\prime}$. We are also going to study the mass and lattice spacing dependence of these splittings arising at higher order in the $\mathrm{W} \chi \mathrm{PT}$ expansion.

The paper is structured as follows: in the next section we collect the $\mathrm{W} \chi \mathrm{PT}$ expressions relating the pion mass-splittings to the Wilson LECs. In section 3 we present the lattice actions used in our study. The determination of the LECs from two of those lattice setups is described in section 4. Finally, a qualitative comparison of the values of these LECs from different choices of the lattice action is reported in section 5 .

\section{Wilson chiral perturbation theory $(\mathrm{W} \chi \mathrm{PT})$}

In this section, we briefly discuss Wilson chiral perturbation theory and introduce the expressions used in our study. For a recent review on the applications of $\chi \mathrm{PT}$ to lattice QCD, we refer to ref. [48].

\footnotetext{
${ }^{1}$ For a recent review, we refer to [37].
} 
Our study will be based on the computation of pseudoscalar meson masses involving Wilson twisted mass fermions. We are therefore interested in a chiral Lagrangian involving a mass matrix $M=m_{0}^{\mathrm{R}}+i \mu_{\ell}^{\mathrm{R}} \tau_{3}$, where $m_{0}^{\mathrm{R}}$ and $\mu_{\ell}^{\mathrm{R}}$ are the renormalised untwisted and twisted quark masses, respectively. The masses $m_{0}$ and $\mu_{\ell}$ appear in the Wilson twisted mass action of eq. (3.2).

At leading order (LO) in the power counting, $m_{0} \sim \mu_{\ell} \sim a^{2} \Lambda_{\mathrm{QCD}}^{3}$, and after a shift of the quark mass to remove a term of $\mathcal{O}(a)$, the partially quenched chiral Lagrangian reads $[10,45]$

$$
\begin{aligned}
\mathcal{L}_{\chi}= & \frac{f^{2}}{8} \operatorname{Str}\left(\partial_{\mu} \Sigma \partial_{\mu} \Sigma^{\dagger}\right)-\frac{f^{2} B_{0}}{4} \operatorname{Str}\left(M^{\dagger} \Sigma+\Sigma^{\dagger} M\right) \\
& -\hat{a}^{2} W_{6}^{\prime}\left[\operatorname{Str}\left(\Sigma+\Sigma^{\dagger}\right)\right]^{2}-\hat{a}^{2} W_{7}^{\prime}\left[\operatorname{Str}\left(\Sigma-\Sigma^{\dagger}\right)\right]^{2} \\
& -\hat{a}^{2} W_{8}^{\prime} \operatorname{Str}\left(\Sigma^{2}+\left[\Sigma^{\dagger}\right]^{2}\right),
\end{aligned}
$$

where $\Sigma$ parametrises the vacuum manifold and thus characterises the Nambu-Goldstone bosons arising from the spontaneous breaking of chiral symmetry. The traces over the flavour indices are denoted by Str and $\hat{a}=2 W_{0} a$. In addition to the continuum LECs $B_{0}$ and $f$ (defined with the convention giving $f_{\pi} \approx 130 \mathrm{MeV}$ ), the chiral Lagrangian also includes $W_{0}, W_{6}^{\prime}, W_{7}^{\prime}$ and $W_{8}^{\prime}$, which are Wilson LECs describing discretisation effects.

In this work we are interested in the determination of the Wilson LECs by matching lattice QCD calculations of pseudoscalar meson masses to their PQW $\chi \mathrm{PT}$ expressions. As already mentioned, we consider Wilson twisted mass fermions at maximal twist. This is achieved in the chiral Lagrangian by setting $m_{0}=0$. At non vanishing values of the lattice spacing, the breaking of flavour symmetry by the twisted mass term in eq. (3.2) implies that the mass of the charged pion $M_{\pi^{ \pm}}$differs from that of the neutral pion $M_{\pi^{0}}$ by $\mathrm{O}\left(a^{2}\right)$ effects. A similar pattern holds for the mass of the "connected neutral pion" mass, $M_{\pi^{(0, \mathrm{c})}}$. The PQW $\chi \mathrm{PT}$ expressions for these three meson masses at LO read [47, 49-51],

$$
\begin{aligned}
M_{\pi^{ \pm}}^{2} & =2 B_{0} \mu_{\ell}, \\
M_{\pi^{0}}^{2} & =2 B_{0} \mu_{\ell}-8 a^{2}\left(2 w_{6}^{\prime}+w_{8}^{\prime}\right), \\
M_{\pi^{(0, \mathrm{c})}}^{2} & =2 B_{0} \mu_{\ell}-8 a^{2} w_{8}^{\prime},
\end{aligned}
$$

where $w_{k}^{\prime}$ is related to the Wilson LEC $W_{k}^{\prime}$ by

$$
w_{k}^{\prime}=\frac{16 W_{0}^{2} W_{k}^{\prime}}{f^{2}} \quad(k=6,8) .
$$

To determine the individual values of the LECs it is, therefore, possible to consider the following mass-splittings:

$$
\begin{aligned}
M_{\pi^{ \pm}}^{2}-M_{\pi^{(0, \mathrm{c})}}^{2} & =8 a^{2} w_{8}^{\prime}, \\
\frac{1}{2}\left(M_{\pi^{(0, \mathrm{c})}}^{2}-M_{\pi^{0}}^{2}\right) & =8 a^{2} w_{6}^{\prime} .
\end{aligned}
$$


From eq. (2.3), it appears that the linear combination of LECs which controls the mass-splitting between charged and neutral pions is given by

$$
c_{2}=-\frac{32 W_{0}^{2}}{f^{2}}\left(2 W_{6}^{\prime}+W_{8}^{\prime}\right) .
$$

This can also be re-expressed as

$$
\begin{aligned}
c_{2} & =\frac{1}{4 a^{2}}\left(M_{\pi^{0}}^{2}-M_{\pi^{ \pm}}^{2}\right), \\
& =-2\left(2 w_{6}^{\prime}+w_{8}^{\prime}\right) .
\end{aligned}
$$

In the $\mathrm{W} \chi \mathrm{PT}$ expressions presented above, two light mass-degenerate flavours were assumed to be present in the sea sector. When considering also other dynamical flavours, such as the strange and the charm quarks, the same expressions hold when assuming that these heavier flavours sufficiently decouple from the light quark sector. In this case, the values of the Wilson LECs will have a further residual dependence on the heavier quark masses.

Before closing this section, we mention that $\mathrm{W} \chi \mathrm{PT}$ calculations at NLO have been carried out for the pion mass and decay constant with $N_{\mathrm{f}}=2$ [52-54] and $N_{\mathrm{f}}=2+1+1$ [55] flavours of twisted mass fermions.

\section{Lattice actions}

The complete lattice action can be written as

$$
S=S_{f}+S_{g},
$$

where $S_{f}$ is the fermionic action and $S_{g}$ is the pure gauge action. As we shall see, in this work we will consider a few alternatives for both the fermionic and the gauge actions in order to explore the dependence of the Wilson LECs on the details of the lattice action. As discussed below, we will use for $S_{F}$ a few variants of Wilson twisted mass fermions.

\subsection{Wilson twisted mass fermions}

The Wilson twisted mass (Wtm) lattice action for the mass degenerate light doublet $(u, d)$ in the so-called twisted basis reads [56, 57],

$$
S_{l}=a^{4} \sum_{x}\left\{\bar{\chi}_{l}(x)\left[D[U]+m_{0, l}+i \mu_{\ell} \gamma_{5} \tau_{3}\right] \chi_{l}(x)\right\}
$$

where $m_{0, l}$ is the untwisted bare quark mass, $\mu_{\ell}$ is the bare twisted light quark mass, $\tau_{3}$ is the third Pauli matrix acting in flavour space and

$$
D[U]=\frac{1}{2}\left[\gamma_{\mu}\left(\nabla_{\mu}+\nabla_{\mu}^{*}\right)-a \nabla_{\mu}^{*} \nabla_{\mu}\right]
$$

is the massless Wilson-Dirac operator. $\nabla_{\mu}$ and $\nabla_{\mu}^{*}$ are the forward and backward gauge covariant difference operators, respectively. Twisted mass light fermions are said to be at 
maximal twist if the bare untwisted mass $m_{0, l}$ is tuned to its critical value, $m_{\text {crit }}$. The quark doublet $\chi_{l}=\left(\chi_{u}, \chi_{d}\right)$ in the twisted basis is related by a chiral rotation to the quark doublet in the physical basis

$$
\psi_{l}^{\text {phys }}=e^{\frac{i}{2} \omega_{l} \gamma_{5} \tau_{3}} \chi_{l}, \quad \bar{\psi}_{l}^{\text {phys }}=\bar{\chi}_{l} e^{\frac{i}{2} \omega_{l} \gamma_{5} \tau_{3}},
$$

where $\omega_{l}$ is the twist angle.

The twisted mass parameter $\mu_{\ell}$ provides an infrared regulator avoiding the presence of accidental zero-modes in the Wilson-Dirac operator. An important property of Wtm fermions is that at maximal twist physical observables are $\mathrm{O}(a)$ improved [57]. In numerical simulations, maximal twist is achieved by tuning the value of the hopping parameter $\kappa=$ $1 /\left(2 m_{0, l}+8\right)$ to its critical value $\kappa_{\text {crit }}$ by tuning the PCAC quark mass $m_{\text {PCAC }}$ to zero. The expected $\mathrm{O}\left(a^{2}\right)$ scaling of physical observables when performing the continuum limit extrapolation has been confirmed in the quenched approximation [58-61] and with $N_{\mathrm{f}}=$ $2[62-65]$ and $N_{\mathrm{f}}=2+1+1[7,66,67]$ dynamical quarks.

A peculiar lattice artifact can appear in observables made out of Wtm quarks due to the breaking of isospin and parity by the twisted mass term in eq. (3.2). This effect, which is expected to vanish in physical quantities at a rate of $\mathrm{O}\left(a^{2}\right)$ when approaching the continuum limit, has been observed to be numerically small in most of the observables which have been analysed [7,62-66]

An exception to this observed small isospin breaking effects is found in the case of the neutral pseudoscalar mass. Indeed, isospin breaking induces a mass-splitting between charged and neutral pion masses. Dedicated numerical studies indicate that while in the charged pion mass only very mild cutoff effects are present, the neutral pion mass is instead affected by significant $\mathcal{O}\left(a^{2}\right)$ effects $[7,62,65,67,68]$. An analysis based on the Symanzik expansion indicates that isospin breaking affects only a limited set of observables in a sizeable way, namely the neutral pion mass and kinematically related quantities [69, 70]. This analysis is complementary to that based on $\mathrm{W} \chi \mathrm{PT}$ where, as previously mentioned, the mass-splitting between charged and neutral pions is parametrised by the combination of LECs appearing in $c_{2}$ defined in eq. (2.8).

The determination of the neutral pion mass $M_{\pi^{0}}$ involves both connected and disconnected contributions. The computation of quark-disconnected diagrams is challenging and requires the employment of specific techniques in order to achieve a statistically significant determination of $M_{\pi^{0}}[71,72]$.

$\mathbf{N}_{\mathrm{f}}=\mathbf{2}+\mathbf{1}+\mathbf{1} \mathbf{W t m}$ fermions. In addition to a doublet of mass-degenerate light quarks $(u, d)$, a heavier doublet with strange and charm quarks - $(s, c)$ - can be incorporated in lattice QCD studied with Wilson twisted mass fermions [73, 74]. Also in this $N_{\mathrm{f}}=2+1+1$ setup, a tuning to maximal twist by imposing $m_{\mathrm{PCAC}}=0$, allows to achieve the automatic $\mathrm{O}(a)$ improvement of physical observables. We refer to ref. [7] for a complete description of the lattice action for the heavier quark doublet and for further details on the lattice setup.

Mixed action with Osterwalder-Seiler valence quarks. Osterwalder-Seiler (OS) valence quarks [75] can be viewed as the building blocks of Wilson twisted mass fermions 
at maximal twist. The OS action for an individual quark flavour $\chi_{f}$ reads

$$
S_{f}^{\mathrm{OS}}=a^{4} \sum_{x}\left\{\bar{\chi}_{f}(x)\left[D[U]+m_{\mathrm{crit}}+i \mu_{f} \gamma_{5} r_{f}\right] \chi_{f}(x)\right\}
$$

where $r_{f}$ (here $\left|r_{f}\right|=1$ ) is the Wilson parameter and $m_{\text {crit }}$ the critical mass. By combining two flavours of OS quarks with opposite signs of $r_{f}$, e.g. $r_{2}=-r_{1}$, the action of a doublet of maximally Wtm fermions of mass $\mu_{f}=\mu_{1}=\mu_{2}$ can be recovered. The benefits of the OS action are that $\mathcal{O}(a)$ improved physical observables [73] can be obtained by using the same estimates of $m_{\text {crit }}$ as in the Wtm case and, thus, avoiding further tuning effort. OS and Wtm fermions coincide with Wilson fermions in the massless limit and consequently share the same renormalisation factors. This simplifies the matching of sea and valence quark masses in the context of a mixed-action with Wtm sea and OS valence quarks.

The pseudoscalar correlation function obtained when considering only the connected contribution to the neutral pion correlation function i.e. when ignoring disconnected diagrams), is precisely the pion correlator with OS fermions. The mass-splittings in eqs. (2.6)-(2.7) can hence be interpreted as involving sea and valence quarks in a mixed action setup.

In this work, we aim at determining the Wilson LECs in a lattice theory with $N_{\mathrm{f}}=$ $2+1+1$ Wtm fermions. A particular effort will be devoted to addressing the main systematic effects present in these determinations. We furthermore aim at exploring the qualitative change on the values of these LECs when varying the details of the lattice action. Below, we briefly summarise the alternative lattice setups used in this work. We will consider variants of the action differing by the presence of smearing of the gauge links in the covariant derivative, the inclusion of the Sheikholeslami-Wohlert term or by a change in the number of dynamical flavours.

Stout smearing. A smearing procedure can be applied to the gauge links entering in the covariant derivatives in eq. (3.3). The stout smearing [76] procedure is analytic in the un-smeared link variables and hence well suited for simulations with the HMC algorithm. The smearing can be iterated several times, with the price of extending the coupling of fermions to the gauge links over a larger region.

Sheikholeslami-Wohlert term. In our comparison of the values of the mass-splittings in eqs. (2.6)-(2.7) from different lattice setups, we will also consider results available in the literature from quenched lattice simulations with Wtm quarks including the SheikholeslamiWohlert term [77].

\subsection{Gauge action}

The lattice gauge actions considered in this work have a generic form which includes a plaquette term $U_{x, \mu, \nu}^{1 \times 1}$ and rectangular $(1 \times 2)$ Wilson loops $U_{x, \mu, \nu}^{1 \times 2}$,

$$
S_{g}=\frac{\beta}{3} \sum_{x}\left(b_{0} \sum_{\substack{\mu, \nu=1 \\ 1 \leq \mu<\nu}}^{4}\left\{1-\operatorname{Re} \operatorname{Tr}\left(U_{x, \mu, \nu}^{1 \times 1}\right)\right\}+b_{1} \sum_{\substack{\mu, \nu=1 \\ \mu \neq \nu}}^{4}\left\{1-\operatorname{Re} \operatorname{Tr}\left(U_{x, \mu, \nu}^{1 \times 2}\right)\right\}\right),
$$


with $\beta=6 / g_{0}^{2}$ the bare inverse coupling and the normalisation condition $b_{0}=1-8 b_{1}$. We will consider the case of the Wilson plaquette [1] action $\left(b_{1}=0\right)$, the tree-level Symanzik improved $[78,79]$ action $\left(b_{1}=-1 / 12\right)$ and the Iwasaki [80-82] action $\left(b_{1}=-0.331\right)$. In Wtm simulations, the strength of the phase transition has been found [21, 22] to depend on the value of the parameter $b_{1}$ in eq. (3.6).

\section{Numerical studies}

\section{1 $\quad N_{\mathrm{f}}=2+1+1 \mathrm{Wtm}$ fermions with Iwasaki gauge action}

The purpose of this study is to determine the mass-splittings in eqs. (2.6) and (2.7), which are directly related to the Wilson LECs $W_{8}^{\prime}$ and $W_{6}^{\prime}$, respectively. The lattice action is composed of the Iwasaki gauge action and $N_{\mathrm{f}}=2+1+1$ flavours of Wilson twisted mass fermions.

The simulations [7] were performed at three values of the lattice gauge coupling, $\beta=$ $1.90,1.95$ and $\beta=2.10$, corresponding to values of the lattice spacing $a \approx 0.09 \mathrm{fm}, 0.08 \mathrm{fm}$ and $0.06 \mathrm{fm}$, respectively. The charged pion mass $M_{\pi^{ \pm}}$approximately ranges from $230 \mathrm{MeV}$ to $510 \mathrm{MeV}$. Simulated volumes correspond to values of $M_{\pi^{ \pm}} L$ larger than 3.3. Physical spatial volumes range from $(1.9 \mathrm{fm})^{3}$ to $(2.8 \mathrm{fm})^{3}$.

The values of the pseudoscalar meson masses for the $N_{\mathrm{f}}=2+1+1$ ensembles are collected in table 1. The mass-splittings in eqs. (2.6)-(2.7), which are directly proportional to $W_{8}^{\prime}$ and $W_{6}^{\prime}$ respectively, are illustrated in figure 1 . We observe that the conditions $W_{8}^{\prime}<0$ and $W_{6}^{\prime}>0$ are fulfilled by the lattice data, in agreement with the bounds derived in refs. $[29,30,35,47]$. In order to quote the values of the Wilson LECs $W_{6,8}^{\prime}$, the systematic effects from quark-mass dependence, residual lattice artifacts and finite volume effects have to be addressed.

In the "large cut-off effects" power counting and at LO in the $\mathrm{W} \chi \mathrm{PT}$ chiral Lagrangian, the mass-splittings in eqs. (2.6)-(2.7) are expected to be independent of the lattice spacing and the light-quark mass. The possible presence of such effects might thus signal effects entering at higher orders in the $\mathrm{W} \chi \mathrm{PT}$ chiral expansion. Since the NLO expressions for these mass-splittings is currently not available in the literature, we rely in our systematic error analysis on a separate study of (i) the continuum-limit of the mass-splittings at a reference mass and (ii) the comparison of a constant and a linear chiral extrapolation in $M_{\pi^{ \pm}}^{2}$.

Starting with point (i), we show in figure 1 the mass splittings eq. (2.6) and (2.7) relevant for $W_{8}^{\prime}$ and $W_{6}^{\prime}$, respectively, as a function of $M_{\pi^{ \pm}}^{2}$. We first observe that data points with similar values of $\left(M_{\pi^{ \pm}} r_{0}\right)^{2}$, but coming from different lattice spacings, tend to be compatible with each other, in particular when considering the larger lattice sizes, represented by the filled symbols. The lattice spacing dependence of the two aforementioned mass splittings at a reference mass $\left(M_{\pi^{ \pm}} r_{0}\right)^{2} \approx 0.55$ is illustrated in figure $2(\mathrm{a})$. Note that only the largest lattice sizes $L$ are considered in this figure. Although the lattice size slightly varies when changing $\beta$, the lattice data fulfils $L \gtrsim 2.5 \mathrm{fm}$ and $M_{\pi^{ \pm}} L \gtrsim 4$ and, therefore, we do not expect a large effect from a small mismatch in the physical volume. Figure 2(a) suggests that the residual lattice spacing effects are small. We remind that these lattice artifacts appear beyond the leading $\mathrm{O}\left(a^{2}\right)$ effects. As an estimate of these 


\begin{tabular}{|lccccccc|}
\hline Ens. & $\beta$ & $L / a$ & $a \mu_{\ell}$ & $a M_{\pi^{ \pm}}$ & $a M_{\pi^{(0, c)}}$ & $a M_{\pi^{0}}$ & $r_{0} / a$ \\
\hline A30.32 & 1.90 & 32 & 0.0030 & $0.1234(03)$ & $0.2111(33)$ & $0.0611(036)$ & $5.23(4)$ \\
A40.32 & & & 0.0040 & $0.1415(04)$ & $0.2274(31)$ & $0.0811(050)$ & \\
A40.24 & & 24 & 0.0040 & $0.1445(06)$ & $0.2375(25)$ & $0.0694(065)$ & \\
A60.24 & & & 0.0060 & $0.1727(06)$ & $0.2544(26)$ & $0.1009(113)$ & \\
A80.24 & & & 0.0080 & $0.1987(06)$ & $0.2659(25)$ & $0.1222(157)$ & \\
A100.24 & & & 0.0100 & $0.2215(04)$ & $0.2883(14)$ & $0.1570(178)$ & \\
\hline A80.24s & 1.90 & 24 & 0.0080 & $0.1982(04)$ & $0.2649(16)$ & $0.1512(115)$ & \\
A100.24s & & & 0.0100 & $0.2215(04)$ & $0.2841(16)$ & $0.1863(141)$ & \\
\hline B25.32 & 1.95 & 32 & 0.0025 & $0.1064(07)$ & $0.1836(21)$ & $0.0605(036)$ & $5.71(4)$ \\
B35.32 & & & 0.0035 & $0.1249(07)$ & $0.1919(17)$ & $0.0710(061)$ & \\
B55.32 & & & 0.0055 & $0.1540(04)$ & $0.2177(19)$ & $0.1323(080)$ & \\
B75.32 & & & 0.0075 & $0.1808(05)$ & $0.2360(12)$ & $0.1557(126)$ & \\
B85.24 & & 24 & 0.0085 & $0.1931(08)$ & $0.2480(11)$ & $0.1879(180)$ & \\
\hline D15.48 & 2.10 & 48 & 0.0015 & $0.0695(03)$ & $0.1124(15)$ & $0.0561(031)$ & $7.46(6)$ \\
D20.48 & & & 0.0020 & $0.0797(05)$ & $0.1170(16)$ & $0.0651(042)$ & \\
D30.48 & & & 0.0030 & $0.0978(04)$ & $0.1296(15)$ & $0.0860(046)$ & \\
\hline D45.32sc & 2.10 & 32 & 0.0045 & $0.1198(05)$ & $0.1480(09)$ & $0.0886(095)$ & \\
\hline
\end{tabular}

Table 1. Determination of the charged $M_{\pi^{ \pm}}$, neutral connected $M_{\pi^{(0, c)}}$ and neutral $M_{\pi^{0}}$ pseudoscalar meson masses from simulations with $N_{\mathrm{f}}=2+1+1$ flavours of Wilson twisted mass fermions at maximal twist and the Iwasaki gauge action. The value of the Sommer scale $r_{0}$ is determined in the chiral limit. The ensemble names containing an "s" or a "c" refer to a change of the strange or the charm quark mass, respectively. The values for $M_{\pi^{ \pm}}$agree within errors with the once published already in ref. [7].

effects, we include in our systematic error analysis the difference between the values of the mass-splittings from the two finer lattice spacings.

Concerning the light-quark mass dependence in point (ii), we can expect that the mass terms appearing at NLO can contain a linear term in $M_{\pi^{ \pm}}^{2}$ but also a term of the form $M_{\pi^{ \pm}}^{2} \log \left(M_{\pi^{ \pm}}^{2}\right)$. Indeed, such terms are present in the $\mathrm{W} \chi \mathrm{PT}$ expression of the mass splitting between the charged and neutral pion masses at NLO [53]. Since the precise form of the these logarithmic terms is yet unknown for the mass-splittings considered here, we limit ourselves to a linear chiral extrapolation in $M_{\pi^{ \pm}}^{2}$. Note that our data is not precise enough to disentangle possible logarithmic contributions. We take as our central values the linearly extrapolated mass-splittings and use the difference with respect to the constant fit as an estimate of the systematic error from the light-quark mass dependence.

Finite volume effects are taken into account by adding to the systematic error the difference between the values of the mass splittings from two ensembles - A40.24 and A40.32 - differing only by a change of lattice size from $L \approx 2.1 \mathrm{fm}$ to $2.8 \mathrm{fm}$. This is expected to be a conservative choice since these ensembles correspond to a rather small 


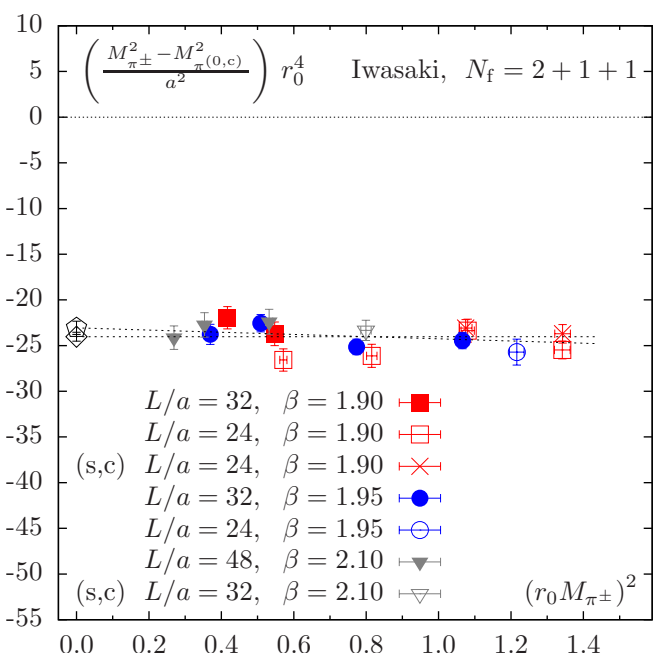

(a)

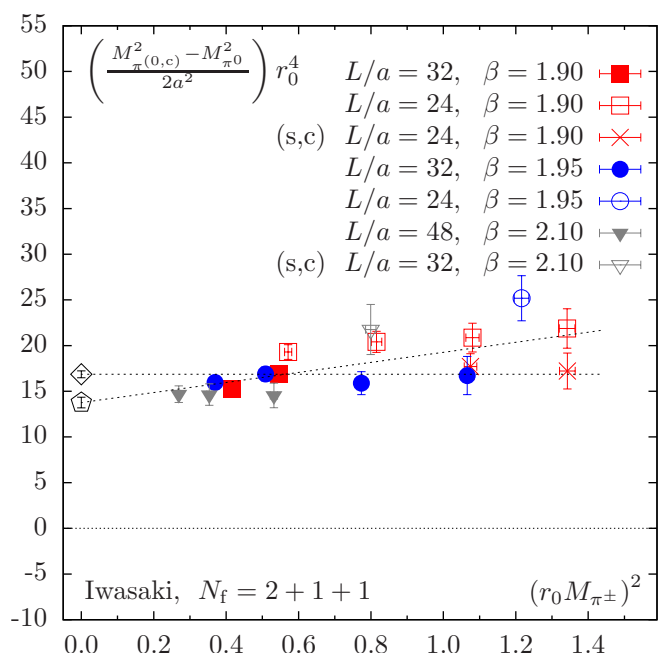

(b)

Figure 1. Determination of the mass-splittings (a) $\left(M_{\pi^{ \pm}}^{2}-M_{\pi^{(0, c)}}^{2}\right) / a^{2}$ in eq. (2.6) and (b) $\left(M_{\pi^{(0, c)}}^{2}-\right.$ $\left.M_{\pi^{0}}^{2}\right) / 2 a^{2}$ in eq. (2.7) as a function of $M_{\pi^{ \pm}}^{2}$. These quantities are in units of the chirally extrapolated Sommer scale $r_{0}$. The mass-splittings are directly related to the Wilson LECs $W_{8}^{\prime}$ and $W_{6}^{\prime}$. The lattice setup with the Iwasaki gauge action and $N_{\mathrm{f}}=2+1+1$ flavours of Wilson twisted mass fermions is considered. Filled and empty symbols signal a change in the lattice size. The label "(s,c)" in the legend indicates the effect of changing the values of the strange and charm quark masses in the sea. The mass-splittings illustrated in these figures assess the size of $\mathrm{O}\left(a^{2}\right)$ discretisation effects. Hence, any lattice spacing dependence in these quantities points to residual higher order discretisation effects. The results of a chiral extrapolation from a constant and a linear fit in $M_{\pi^{ \pm}}^{2}$ are shown. The deviation between the two extrapolated values is included in the systematic error analysis.

light-quark mass - and therefore finite size effects can be non-negligible. Also, these ensembles were obtained at the coarsest lattice spacing, where possible finite size effects (FSE) from the neutral pion mass should be larger.

As already mentioned, the determination of the Wilson LECs $W_{6,8}^{\prime}$ from lattice data with $N_{\mathrm{f}}=2+1+1$ flavours assumes that the strange and the charm sea-quarks decouple sufficiently from the light-quark dynamics. The residual heavy quark mass dependence present in $W_{6,8}^{\prime}$ can be studied by varying the strange and charm quark masses in the neighbourhood of their physical values. This effect is illustrated in figure 1 by the points labelled by "(s,c)" in the legend. We use the difference between the mass-splittings from ensembles A80.24 and A80.24s - which only differ by a change of the strange and charm quark masses - to estimate this systematic effect. We expect that this is a conservative choice because (a) the ensemble A $80.24 \mathrm{~s}$ has a strange quark mass which is very close to the physical point, (b) the change in the strange quark mass is largest for A80.24 and A80.24s and (c) the effect of strange sea-quarks should be larger than that of charm quarks.

After combining the previously discussed systematic uncertainties in quadrature, we obtain the following values for the mass-splittings for the case of a lattice setup with 


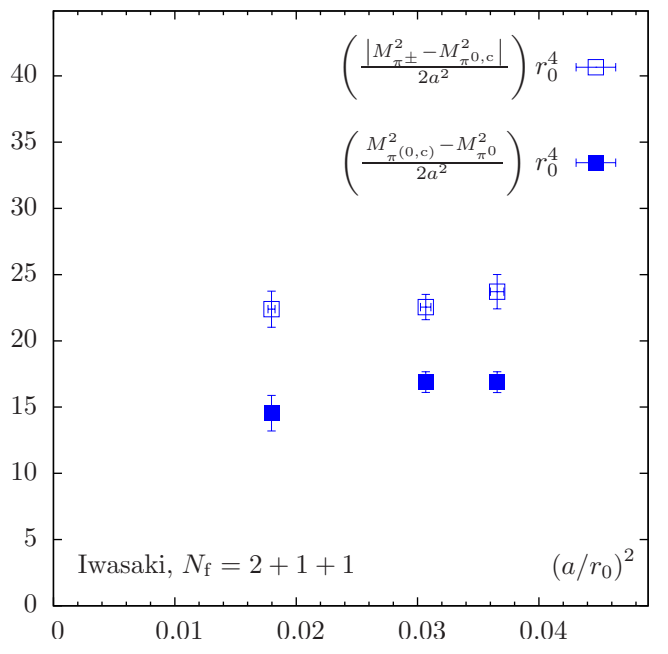

(a)

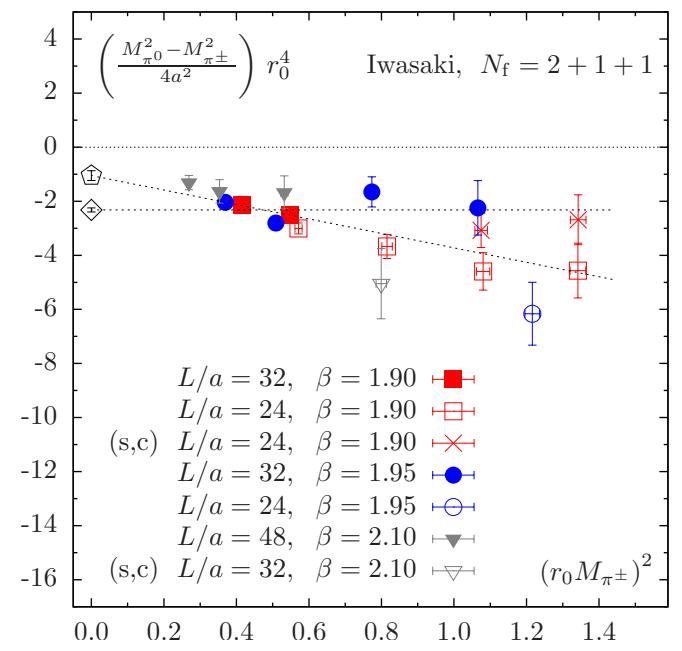

(b)

Figure 2. (a) Lattice spacing dependence of the mass-splittings $\left|M_{\pi^{ \pm}}^{2}-M_{\pi^{(0, \mathrm{c})}}^{2}\right| / a^{2}-$ empty symbols - and $\left(M_{\pi^{(0, c)}}^{2}-M_{\pi^{0}}^{2}\right) / 2 a^{2}$ - filled symbols - at a reference mass $\left(M_{\pi^{ \pm}} r_{0}\right)^{2} \approx 0.55$. For a better visibility, the absolute value of the mass splitting is considered in the case of the empty symbols. (b) Pion mass-splitting, $\left(M_{\pi^{0}}^{2}-M_{\pi^{ \pm}}^{2}\right) / a^{2}$, normalised according to eq. (2.9) in order to relate it to the combination of Wilson LECs $c_{2}$. The chiral extrapolation using a constant and a linear fit in $M_{\pi^{ \pm}}^{2}$ is also shown. The lattice setup with the Iwasaki gauge action and $N_{\mathrm{f}}=2+1+1$ flavours of Wilson twisted mass fermions is considered.

$N_{\mathrm{f}}=2+1+1 \mathrm{Wtm}$ fermions and the Iwasaki gauge action,

$$
\begin{aligned}
& \left(\frac{M_{\pi^{ \pm}}^{2}-M_{\pi^{(0, \mathrm{c})}}^{2}}{a^{2}}\right) r_{0}^{4}=-23.0 \pm 0.7 \pm 3.0 \\
& \left(\frac{M_{\pi^{(0, \mathrm{c})}}^{2}-M_{\pi^{0}}^{2}}{2 a^{2}}\right) r_{0}^{4}=+13.8 \pm 0.6 \pm 5.6
\end{aligned}
$$

where the first error is statistical and the second systematic. The corresponding values of the Wilson LECs are collected in table 2. As already anticipated, the results for $w_{6,8}^{\prime}$ are precise enough to identify a definite sign for these LECs.

The combination of LECs $c_{2}$, can be determined directly from the mass-splitting $M_{\pi^{0}}^{2}-$ $M_{\pi^{ \pm}}^{2}$ as indicated in eq. (2.9). The measurements of this mass-splitting are illustrated in figure 2(b) where the results of a chiral extrapolation by using a constant and a linear fit in $M_{\pi^{ \pm}}^{2}$ are also shown. For the case of $c_{2}$, we provide in table 2 the results from both these chiral extrapolations and quote in the individual numbers only the statistical error. The values arising from these extrapolations are both compatible with a negative sign of $c_{2}$.

The $\mathrm{W} \chi \mathrm{PT}$ expressions at NLO relevant for $c_{2}$ have been derived in ref [53]. In addition to the Wilson LECs appearing at LO and to the usual Gasser-Leutwyler LECs, other parameters also appear at NLO. A complete determination of these parameters is beyond the scope of this study. We postpone such an analysis to a future dedicated study of the 


\begin{tabular}{|cccc|}
\hline & $w_{8}^{\prime} r_{0}^{4}$ & $w_{8}^{\prime}$ & $W_{8}^{\prime}\left(r_{0}^{6} W_{0}^{2}\right)$ \\
syst. & $-2.9(4)$ & $-[571(32) \mathrm{MeV}]^{4}$ & $-0.0138(22)$ \\
\hline & $w_{6}^{\prime} r_{0}^{4}$ & $w_{6}^{\prime}$ & $W_{6}^{\prime}\left(r_{0}^{6} W_{0}^{2}\right)$ \\
syst. & $+1.7(7)$ & $+[502(58) \mathrm{MeV}]^{4}$ & $+0.0082(34)$ \\
\hline & $c_{2} r_{0}^{4}$ & $c_{2}$ & $-2\left(2 W_{6}^{\prime}+W_{8}^{\prime}\right)\left(r_{0}^{6} W_{0}^{2}\right)$ \\
lin. & $-1.1(2)$ & $-[444(28) \mathrm{MeV}]^{4}$ & $-0.0050(10)$ \\
cst. & $-2.3(1)$ & $-[541(24) \mathrm{MeV}]^{4}$ & $-0.0111(10)$ \\
\hline
\end{tabular}

Table 2. Determination of the Wilson LECs $W_{6,8}^{\prime}\left(w_{6,8}^{\prime}\right)$ and $c_{2}$ from a lattice setup with $N_{\mathrm{f}}=$ $2+1+1 \mathrm{Wtm}$ fermions and the Iwasaki gauge action. For the values quoted in physical units, the input $r_{0}=0.45(2) \mathrm{fm}$ has been used. The values in the last column derive from eq. (2.5), where the input $r_{0} f=0.276(12)$ from [7] has been used. For the case of $W_{6,8}^{\prime}$ the systematic error analysis described in the text has been incorporated in the overall uncertainty indicated by the label "syst." in the table. For $c_{2}$, in the last block, we quote separately the results of a constant and a linear chiral extrapolation in $M_{\pi^{ \pm}}^{2}$ - as illustrated in figure 2(b) - and the quoted errors are purely statistical.

$\mathrm{W} \chi \mathrm{PT}$ description of both the pion mass and decay constant. First results for the GasserLeutwyler LECs, from fits based on continuum $\chi \mathrm{PT}$, have been presented in refs. [7, 83].

The connected neutral pion mass, $M_{\pi^{(0, c)}}$, is an important ingredient in order to isolate the individual values of the LECs $W_{6,8}^{\prime}$. As already pointed out, the connected neutral pion can be interpreted as the pion of a mixed action with OS fermions. Such a mixed action has been used to determine observables in the Kaon sector $[67,84,85]$. We note that extensions of the analytical expressions to $\mathrm{SU}(3) \mathrm{W} \chi \mathrm{PT}$ is currently not available in the literature. Contrary to the pion case, the absence of disconnected diagrams in correlations functions in the Kaon sector could possibly allow to consider quantities from which the Wilson LECs can be determined with good accuracy.

\section{2 $N_{\mathrm{f}}=2 \mathrm{Wtm}$ fermions with tlSym gauge action}

In this section, we again determine the LECs $W_{6,8}^{\prime}$, but this time using $N_{\mathrm{f}}=2$ flavours of Wilson twisted mass fermions and the tree-level Symanzik improved gauge action [3, 65, 72]. We already anticipate that a smaller set of ensembles and of measurements of the relevant pion masses are available in this case, in comparison to the $N_{\mathrm{f}}=2+1+1$ case discussed previously. Therefore, the resulting determinations and comparisons might suffer from insufficient control of systematic effects. However, we think that already a qualitative comparison can provide useful information to parametrise the size of cutoff effects from different lattice setups.

The simulations considered in this work $[65,87]$ were performed at three values of the lattice gauge coupling $\beta=3.90,4.05$ and $\beta=4.20$, corresponding to values of the lattice spacing $a \approx 0.08 \mathrm{fm}, 0.07 \mathrm{fm}$ and $0.05 \mathrm{fm}$, respectively. The charged pion mass $M_{\pi^{ \pm}}$approximately ranges from $310 \mathrm{MeV}$ to $460 \mathrm{MeV}$. Physical spatial volumes range from $(1.3 \mathrm{fm})^{3}$ to $(2.6 \mathrm{fm})^{3}$ and ensembles which differ only by the lattice size have been considered in order to address the size of finite volume effects in the determination of the Wilson LECs. 


\begin{tabular}{|ccccccc|}
\hline$\beta$ & $L / a$ & $a \mu_{\ell}$ & $a M_{\pi^{ \pm}}$ & $a M_{\pi^{(0, \mathrm{c})}}$ & $a M_{\pi^{0}}$ & $r_{0} / a$ \\
\hline 3.90 & 32 & 0.0040 & $0.1338(02)$ & $0.2080(30)$ & $0.1100(080)$ & $5.35(4)$ \\
& 24 & 0.0040 & $0.1362(07)$ & $0.2120(30)$ & $0.1090(070)$ & \\
& 16 & 0.0040 & $0.1596(30)$ & $0.2226(95)$ & - & \\
& 24 & 0.0064 & $0.1694(04)$ & - & $0.1340(100)$ & \\
& & 0.0085 & $0.1940(05)$ & - & $0.1690(110)$ & \\
& 16 & 0.0074 & $0.1963(17)$ & $0.2541(55)$ & - & \\
\hline 4.05 & 32 & 0.0030 & $0.1038(06)$ & $0.1500(30)$ & $0.0900(060)$ & $6.71(4)$ \\
& 20 & 0.0030 & $0.1191(41)$ & $0.1571(62)$ & - & \\
& 32 & 0.0060 & $0.1432(06)$ & $0.1800(20)$ & $0.1230(060)$ & \\
\hline 4.20 & 24 & 0.0020 & $0.0941(31)$ & $0.1157(61)$ & - & $8.36(6)$ \\
\hline
\end{tabular}

Table 3. Determination of the charged $M_{\pi^{ \pm}}$, neutral connected $M_{\pi^{(0, c)}}$ and neutral $M_{\pi^{0}}$ pseudoscalar meson masses from simulations with $N_{\mathrm{f}}=2$ flavours of Wilson twisted mass fermions at maximal twist and the tree-level Symanzik improved gauge action [65]. The value of the Sommer scale $r_{0}$ is determined in the chiral limit [86].

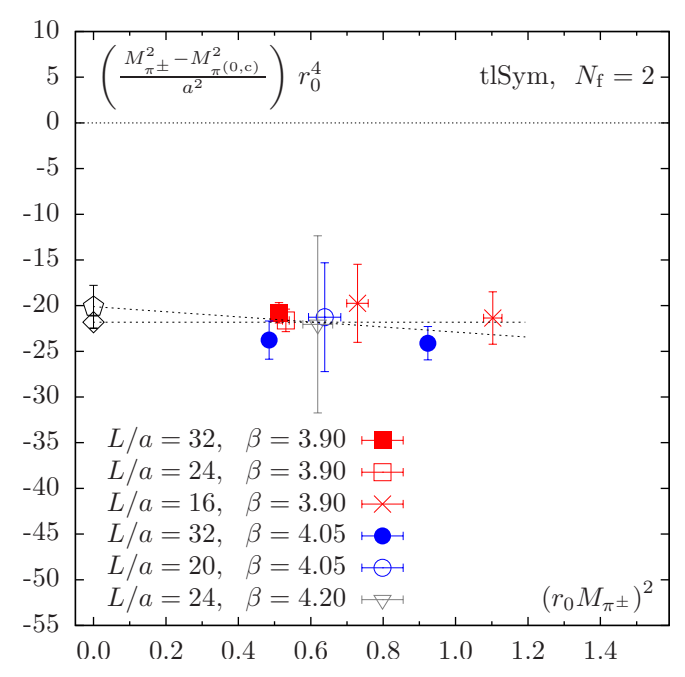

(a)

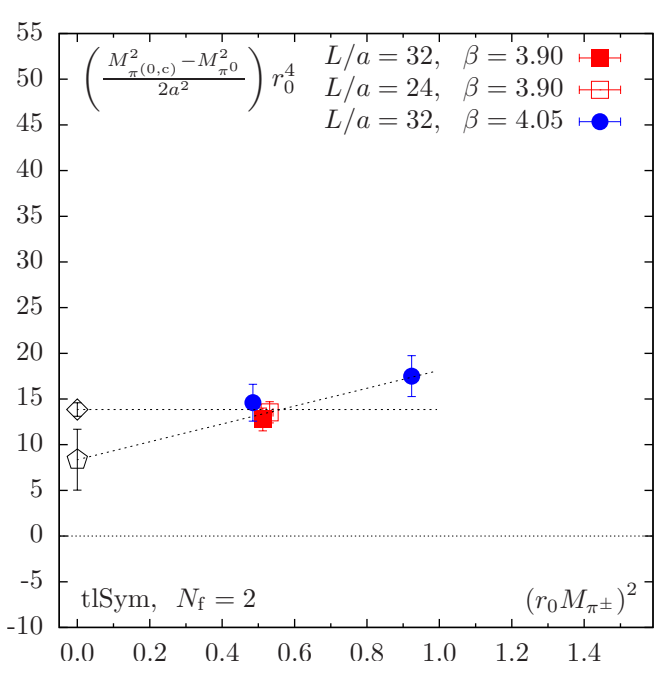

(b)

Figure 3. Determination of the mass-splittings (a) $\left(M_{\pi^{ \pm}}^{2}-M_{\pi^{(0, c)}}^{2}\right) / a^{2}$ in eq. (2.6) and (b) $\left(M_{\pi^{(0, c)}}^{2}-\right.$ $\left.M_{\pi^{0}}^{2}\right) / 2 a^{2}$ in eq. (2.7) as a function of $M_{\pi^{ \pm}}^{2}$. These quantities are in units of the chirally extrapolated Sommer scale $r_{0}$. The mass-splittings are directly related to the Wilson LECs $W_{8}^{\prime}$ and $W_{6}^{\prime}$. The lattice setup with the tree-level Symanzik improved (tlSym) gauge action and $N_{\mathrm{f}}=2$ flavours of Wilson twisted mass fermions is considered. Filled and empty symbols signal a change in the lattice size.

The values of the pseudoscalar meson masses [65] for the $N_{\mathrm{f}}=2$ ensembles are collected in table 3. The mass-splittings in eqs. (2.6)-(2.7) are illustrated in figure 3. In order to explore the systematic effects present in these determinations, we follow a similar path to that described for the case of $N_{\mathrm{f}}=2+1+1$ ensembles. The availability of ensembles differing 


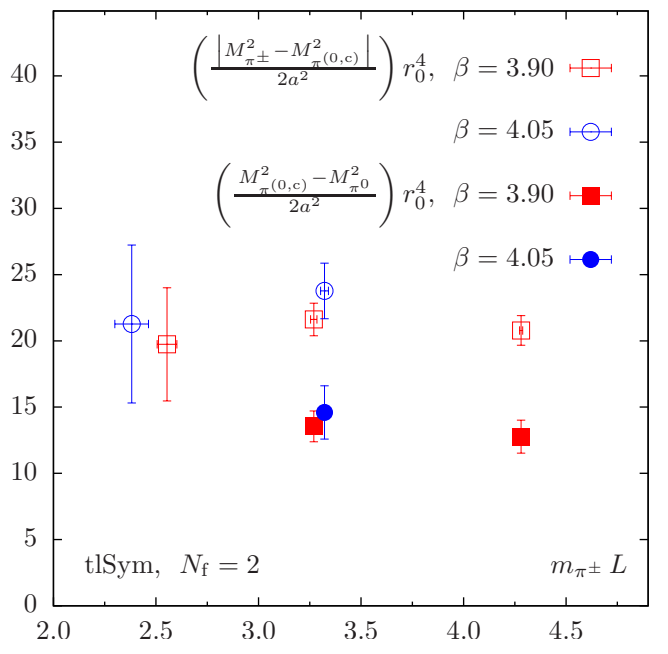

(a)

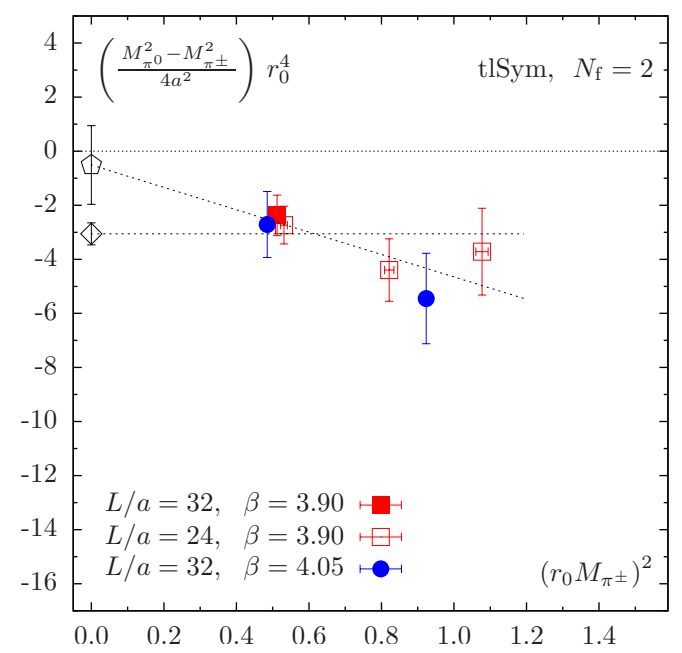

(b)

Figure 4. (a) Finite volume effects on the mass-splittings $\left|M_{\pi^{ \pm}}^{2}-M_{\pi^{(0, c)}}^{2}\right| / a^{2}-$ empty symbols and $\left(M_{\pi^{(0, c)}}^{2}-M_{\pi^{0}}^{2}\right) / 2 a^{2}$ - filled symbols. Note that the absolute value of the mass-splitting is used in the case of empty symbols. (b) Pion mass-splitting, $\left(M_{\pi^{0}}^{2}-M_{\pi^{ \pm}}^{2}\right) / a^{2}$, normalised according to eq. (2.9) in order to relate it to the combination of Wilson LECs $c_{2}$. The chiral extrapolation using a constant and a linear fit in $M_{\pi^{ \pm}}^{2}$ is also shown. The lattice setup with the tree-level Symanzik improved (tlSym) gauge action and $N_{\mathrm{f}}=2$ flavours of Wilson twisted mass fermions is considered.

only by the physical volume allows to address the size of FSE in the mass-splittings. This is illustrated in figure 4 (a). At $\beta=3.90$, a set of three ensembles with $L / a=16,24$ and 32 could be used for the case of $\left(M_{\pi^{ \pm}}^{2}-M_{\pi^{(0, \mathrm{c})}}^{2}\right) / a^{2}$. With the current statistical uncertainties, no clear signs of FSE can be observed in the data. Furthermore, data from two different lattice spacings $-\beta=3.90$ and 4.05 - agree within errors, indicating the absence of large residual lattice artifacts in these mass-splittings. However, the lack of sufficient data does not allow to address the mass dependence of the mass-splitting $\left(M_{\pi^{(0, \mathrm{c})}}^{2}-M_{\pi^{0}}^{2}\right) / a^{2}$ in a satisfactory way. In analogy to the $N_{\mathrm{f}}=2+1+1$ case, we include the deviation between a constant and a linear extrapolation in $M_{\pi^{ \pm}}^{2}$ in the estimate of the systematic uncertainties. The central value is taken from the result of the linear fit.

For the case of a lattice setup with $N_{\mathrm{f}}=2 \mathrm{Wtm}$ fermions at maximal twist and the tlSym gauge action, we obtain the following values for the mass-splittings

$$
\begin{aligned}
& \left(\frac{M_{\pi^{ \pm}}^{2}-M_{\pi^{(0, \mathrm{c})}}^{2}}{a^{2}}\right) r_{0}^{4}=-20.1 \pm 2.3 \pm 1.7, \\
& \left(\frac{M_{\pi^{(0, \mathrm{c})}}^{2}-M_{\pi^{0}}^{2}}{2 a^{2}}\right) r_{0}^{4}=+8.4 \pm 3.3 \pm 5.5,
\end{aligned}
$$

where the first error is statistical and the second systematic. The corresponding values of the Wilson LECs are collected in table 4 . The LEC $W_{8}^{\prime}$ has recently been determined from a mixed action involving the same $N_{\mathrm{f}}=2$ lattice action in the sea sector as that de- 


\begin{tabular}{|ccc|}
\hline$w_{8}^{\prime} r_{0}^{4}$ & $w_{8}^{\prime}$ & $W_{8}^{\prime}\left(r_{0}^{6} W_{0}^{2}\right)$ \\
$-2.5(4)$ & $-[552(025) \mathrm{MeV}]^{4}$ & $-0.0119(17)$ \\
\hline$w_{6}^{\prime} r_{0}^{4}$ & $w_{6}^{\prime}$ & $W_{6}^{\prime}\left(r_{0}^{6} W_{0}^{2}\right)$ \\
$+1.0(8)$ & $+[443(138) \mathrm{MeV}]^{4}$ & $+0.0049(38)$ \\
\hline
\end{tabular}

Table 4. Determination of the Wilson LECs $W_{6,8}^{\prime}\left(w_{6,8}^{\prime}\right)$ from a lattice setup with $N_{\mathrm{f}}=2 \mathrm{Wtm}$ fermions and the tlSym gauge action. For the values quoted in physical units, the input $r_{0}=$ $0.45(2) \mathrm{fm}$ has been used. The values in the last column derive from eq. (2.5), where the input $r_{0} f=0.275(6)$ from [65] has been used.

scribed here, but with Neuberger overlap valence quark [46]. The value quoted in ref. [46], $W_{8}^{\prime}\left(r_{0}^{6} W_{0}^{2}\right)=-0.0064(24)$, differs from the estimate in table 4 at the 2 -sigma level. However, we stress once more that our present $N_{\mathrm{f}}=2$ estimate does not include a complete assessment of the systematic errors.

The determination of $c_{2}$ from $N_{\mathrm{f}}=2$ ensembles is illustrated in figure 4(b). More data would be needed to isolate the residual mass-dependence present in $c_{2}$. For this reason we opt for quoting separately the results of a constant and a linear chiral extrapolation in $M_{\pi^{ \pm}}^{2}$

$$
\begin{aligned}
& c_{2} r_{0}^{4} \text { [cst. }=-3.1 \pm 0.4, \\
& c_{2} r_{0}^{4}[\text { lin. }]=-0.5 \pm 1.5,
\end{aligned}
$$

where the errors are statistical only. These values are consistent with those arising from the measurements of pseudoscalar meson masses in ref. [65] and are also very similar to the result obtained in ref. [52] for $K=-4 c_{2}$ from twisted mass finite volume effects. All the lattice measurements favour a negative sign of $c_{2}$. However, as already mentioned, more data would be needed to properly address the residual mass dependence.

We refer to refs. $[65,86]$ for more details about the description of the pion mass and decay constant by means of $\chi \mathrm{PT}$ expressions including discretisation effects.

\section{Discussion}

In this section we collect a few comments concerning the extraction of the Wilson LECs. The LECs $W_{6}^{\prime}$ and $c_{2}$ depend on the neutral pion mass $M_{\pi^{0}}$. The statistical error in $M_{\pi^{0}}$ is dominated by the contribution of disconnected diagrams. ${ }^{2}$ We observe that the precise form of the light-quark mass dependence of the mass-splittings related to $W_{6}^{\prime}$ and $c_{2}-$ see e.g. figures 1(b) and 2(b) - cannot be addressed within the present uncertainties. As previously discussed this mass dependence can arise at NLO in the $\mathrm{W} \chi \mathrm{PT}$ expansion. We can, however, not exclude at this stage that this higher-order effects are negligible. This issue is particularly relevant for the case of $c_{2} \propto-\left(2 w_{6}^{\prime}+w_{8}^{\prime}\right)$, where a partial cancellation of

\footnotetext{
${ }^{2}$ It is interesting to note that table 1 indicates that the relative error on the neutral pion mass is roughly independent of the light-quark mass and that it decreases when increasing the volume. In practice, in the current simulation conditions, this implies that the measurements of $M_{\pi^{0}}$ are statistically more precise for the ensembles with lighter quark masses.
} 


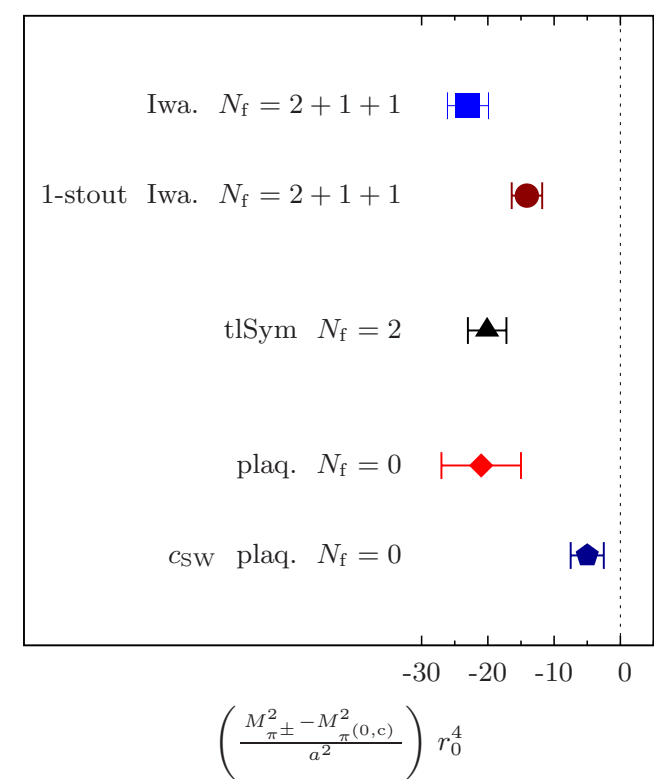

(a)

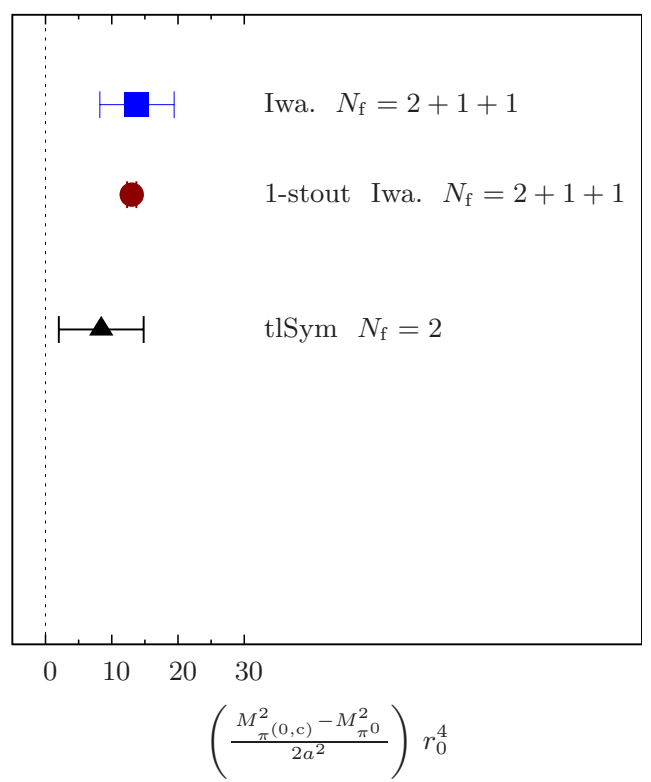

(b)

Figure 5. Comparison of the values of the mass-splittings (a) $\left(M_{\pi^{ \pm}}^{2}-M_{\pi^{(0, c)}}^{2}\right) / a^{2}$ and (b) $\left(M_{\pi^{(0, c)}}^{2}-\right.$ $\left.M_{\pi^{0}}^{2}\right) / 2 a^{2}$ from different lattice setups (see text for details).

the effect of $w_{6}^{\prime}$ and $w_{8}^{\prime}$ is present. The presence of higher order effects in the determination of $c_{2}$ has also been discussed in ref. [42]. We recall that the sign of $c_{2}$ controls the appearance of an Aoki $\left(c_{2}>0\right)$ or of a Sharpe-Singleton $\left(c_{2}<0\right)$ scenario for the phase structure of Wilson fermions.

From the previously discussed determinations, a comparison of the values of the Wilson LECs from the lattice actions (i) $N_{\mathrm{f}}=2+1+1$ flavours of Wtm fermions at maximal twist and Iwasaki gauge action and (ii) $N_{\mathrm{f}}=2$ flavours of Wtm quarks and tlSym gauge action, suggests that $W_{6,8}^{\prime}$ and $c_{2}$ do not vary significantly in between these two setups. Let us extend this observation by performing a comparison of the pion mass-splittings as determined from different lattice actions. We stress that a complete assessment of the overall uncertainty is not available for most of these measurements and therefore the comparison remains at the qualitative level.

Lattice simulations with $N_{\mathrm{f}}=2+1+1 \mathrm{Wtm}$ fermions and the Iwasaki gauge action, but including in addition one iteration of stout smearing - labelled 1-stout - have been reported in ref. [7]. A qualitative comparison of the effect of the stout smearing on the size of the mass-splittings is shown in figure 5. Note that a single ensemble is used in the estimate of the mass-splittings for the case of stout smearing. The used smearing seems to help in reducing the magnitude of the splitting $\left(M_{\pi^{ \pm}}^{2}-M_{\pi^{(0, \mathrm{c})}}^{2}\right) / a^{2}$ while - given the current uncertainties -it does not introduce a significant change in $\left(M_{\pi^{(0, \mathrm{c})}}^{2}-M_{\pi^{0}}^{2}\right) / a^{2}$.

Figure 5(a) also includes the estimates of the mass-splitting $\left(M_{\pi^{ \pm}}^{2}-M_{\pi^{(0, \mathrm{c})}}^{2}\right) / a^{2}$ as determined from quenched ensembles either with or without the presence of the SheikholeslamiWohlert term. Maximally twisted-mass fermions and the plaquette gauge action are used 
in both cases. The values of the mass-splitting are derived from studies available in the literature. For the case without the Sheikholeslami-Wohlert term, results are taken from ref. [68]. We use the information from different lattice spacings and quark masses to estimate the size of the systematic effects in $\left(M_{\pi^{ \pm}}^{2}-M_{\pi^{(0, \mathrm{c})}}^{2}\right) / a^{2}$. For the case in which the Sheikholeslami-Wohlert term is included - labelled $c_{\mathrm{SW}}$ - we follow ref. [88], where the non-perturbative determination of $c_{\mathrm{SW}}$ was used.

For cases other than those involving the Sheikholeslami-Wohlert term or stoutsmearing, figure 5(a) suggests that, with the current precision, the value of the masssplitting $\left(M_{\pi^{ \pm}}^{2}-M_{\pi^{(0, c)}}^{2}\right) / a^{2}$ does not significantly depend on a simultaneous change of the number of flavours $N_{\mathrm{f}}$ and of the gauge action. Note however that we cannot exclude that a change only in $N_{\mathrm{f}}$ or only in the parameter $b_{1}$ of the gauge action, leads to a different conclusion. It would be very desirable, if further actions are investigated and the precision could be increased.

One important observation arising from the measurements in ref. [88] is that the introduction of the Sheikholeslami-Wohlert term in quenched studies significantly reduces the size of $\mathrm{O}\left(a^{2}\right)$ effects by lowering the value of the mass-splitting $\left(M_{\pi^{ \pm}}^{2}-M_{\pi^{(0, \mathrm{c})}}^{2}\right) / a^{2}$. It would thus be interesting to study whether this result still holds for simulations with dynamical fermions and whether the value of $\left(M_{\pi^{(0, c)}}^{2}-M_{\pi^{0}}^{2}\right) / 2 a^{2}$ is also reduced in that case.

\section{Conclusions}

We have presented the determination of the Wilson LECs $W_{6,8}^{\prime}$ and $c_{2}$, parametrising the size of $\mathrm{O}\left(a^{2}\right)$ lattice artifacts in $\mathrm{W} \chi \mathrm{PT}$, from simulations with a lattice action composed out of the Iwasaki gauge action and $N_{\mathrm{f}}=2+1+1$ flavours of Wilson twisted mass fermions at maximal twist. The values of $W_{6,8}^{\prime}$ include a rather complete account of the systematic uncertainties. Our measurements satisfy the recently derived bounds [29, 30, 35, 47], $W_{8}^{\prime}<0$ and $W_{6}^{\prime}>0$.

We have also explored the dependence of the mass-splittings $\left(M_{\pi^{ \pm}}^{2}-M_{\pi^{(0, c)}}^{2}\right) / a^{2}$ and $\left(M_{\pi^{(0, \mathrm{c})}}^{2}-M_{\pi^{0}}^{2}\right) / 2 a^{2}$ on the choice of the lattice action. From this qualitative comparison, it is tempting to conjecture that a lattice action made of dynamical twisted mass fermions including the Sheikholeslami-Wohlert and smearing might lead to a reduction of the masssplitting $\left(M_{\pi^{ \pm}}^{2}-M_{\pi^{(0, c)}}^{2}\right) / a^{2}$. Further studies are needed to clarify this point and to extend it to the case of $\left(M_{\pi^{(0, \mathrm{c})}}^{2}-M_{\pi^{0}}^{2}\right) / 2 a^{2}$.

A partial cancellation of the contributions from $W_{6}^{\prime}$ and $W_{8}^{\prime}$ implies that the residual mass-dependence of $c_{2}$ is more sensitive to higher order effects in the $\mathrm{W} \chi \mathrm{PT}$ expansion. While this potential reduction of $c_{2}$ would certainly be beneficial, the precise determination of its value cannot be achieved with the currently available data.

The determination of the Wilson LECs can help to quantify the size of $\mathrm{O}\left(a^{2}\right)$ terms in a given lattice action. Knowing these LECs, in particular with better precision, can significantly contribute to design a lattice fermion action with small lattice artifacts, thus allowing to reach the continuum limit in a better controlled way. In addition, an independent calculation of the Wilson LECs, as carried through here, can be used in chiral perturbation theory fits of light meson observables by constraining these fits to lattice data. Thus we 
think that the study of the (connected and full) neutral and charged pion masses of this work can be beneficial for many other groups working with Wilson-like lattice fermions.

\section{Acknowledgments}

We thank Giancarlo Rossi for useful comments on the manuscript. The computer time for this project was made available to us by the John von Neumann-Institute for Computing (NIC) on the JUDGE and Jugene systems in Jülich and the IDRIS (CNRS) computing center in Orsay. In particular we thank U.-G. Meißner for granting us access on JUDGE. Falk Zimmermann cross-checked correlation functions for one of our ensembles, which we gratefully acknowledge. G. H. acknowledges the support by DFG (SFB 1044), the Spanish Ministry for Education and Science project FPA2009-09017, the Consolider-Ingenio 2010 Programme CPAN (CSD2007-00042), the Comunidad Autónoma de Madrid (HEPHACOS P-ESP-00346 and HEPHACOS S2009/ESP-1473) and the European project STRONGnet (PITN-GA-2009-238353). K. J. was supported in part by the Cyprus Research Promotion Foundation under contract ПРО $\Sigma$ E $\Lambda$ KY $\Sigma H / E M \Pi E I P O \Sigma / 0311 / 16$. This work has been supported in part by the DFG Sonderforschungsbereich/ Transregio SFB/TR9. Two of the authors (K. O. and C. U.) were supported by the Bonn-Cologne Graduate School (BCGS) of Physics and Astronomie. This project was supported in parts by the DFG in SFB/TR16 and CRC 110.

Open Access. This article is distributed under the terms of the Creative Commons Attribution License which permits any use, distribution and reproduction in any medium, provided the original author(s) and source are credited.

\section{References}

[1] K.G. Wilson, Confinement of quarks, Phys. Rev. D 10 (1974) 2445 [inSPIRE].

[2] L. Del Debbio, L. Giusti, M. Lüscher, R. Petronzio and N. Tantalo, QCD with light Wilson quarks on fine lattices (I): first experiences and physics results, JHEP 02 (2007) 056 [hep-lat/0610059] [INSPIRE].

[3] ETM collaboration, P. Boucaud et al., Dynamical twisted mass fermions with light quarks, Phys. Lett. B 650 (2007) 304 [hep-lat/0701012] [INSPIRE].

[4] Hadron Spectrum collaboration, H.-W. Lin et al., First results from 2+1 dynamical quark flavors on an anisotropic lattice: Light-hadron spectroscopy and setting the strange-quark mass, Phys. Rev. D 79 (2009) 034502 [arXiv:0810.3588] [INSPIRE].

[5] BMW collaboration, S. Dürr et al., Ab-Initio Determination of Light Hadron Masses, Science 322 (2008) 1224 [arXiv:0906.3599] [INSPIRE].

[6] PACS-CS collaboration, S. Aoki et al., Physical Point Simulation in 2+1 Flavor Lattice QCD, Phys. Rev. D 81 (2010) 074503 [arXiv:0911.2561] [inSPIRE].

[7] ETM collaboration, R. Baron et al., Light hadrons from lattice QCD with light (u,d), strange and charm dynamical quarks, JHEP 06 (2010) 111 [arXiv:1004.5284] [INSPIRE].

[8] BMW collaboration, S. Dürr et al., Lattice QCD at the physical point: Simulation and analysis details, JHEP 08 (2011) 148 [arXiv:1011.2711] [INSPIRE]. 
[9] QCDSF-UKQCD collaboration, W. Bietenholz et al., Flavour blindness and patterns of flavour symmetry breaking in lattice simulations of up, down and strange quarks, Phys. Rev. D 84 (2011) 054509 [arXiv: 1102.5300] [INSPIRE].

[10] S.R. Sharpe and J. Singleton, Robert L., Spontaneous flavor and parity breaking with Wilson fermions, Phys. Rev. D 58 (1998) 074501 [hep-lat/9804028] [INSPIRE].

[11] S. Aoki, New Phase Structure for Lattice QCD with Wilson Fermions, Phys. Rev. D 30 (1984) 2653 [INSPIRE].

[12] M. Creutz, Wilson fermions at finite temperature, hep-lat/9608024 [INSPIRE].

[13] S. Aoki and A. Gocksch, Spontaneous breaking of flavor symmetry and parity in lattice QCD with Wilson fermions, Phys. Rev. D 45 (1992) 3845 [INSPIRE].

[14] T. Blum et al., QCD thermodynamics with Wilson quarks at large kappa, Phys. Rev. D 50 (1994) 3377 [hep-lat/9404006] [INSPIRE].

[15] S. Aoki, On the phase structure of QCD with Wilson fermions, Prog. Theor. Phys. Suppl. 122 (1996) 179 [hep-lat/9509008] [INSPIRE].

[16] S. Aoki, T. Kaneda and A. Ukawa, Structure of critical lines in quenched lattice QCD with the Wilson quark action, Phys. Rev. D 56 (1997) 1808 [hep-lat/9612019] [INSPIRE].

[17] JLQCD collaboration, S. Aoki et al., Nontrivial phase structure of $N_{f}=3$ QCD with $O(a)$ improved Wilson fermion at zero temperature, Nucl. Phys. Proc. Suppl. 106 (2002) 263 [hep-lat/0110088] [INSPIRE].

[18] E.-M. Ilgenfritz, W. Kerler, M. Muller-Preussker, A. Sternbeck and H. Stuben, A Numerical reinvestigation of the Aoki phase with $N_{f}=2$ Wilson fermions at zero temperature, Phys. Rev. D 69 (2004) 074511 [hep-lat/0309057] [INSPIRE].

[19] JLQCD collaboration, S. Aoki et al., Bulk first-order phase transition in three-flavor lattice QCD with $O($ a)-improved Wilson fermion action at zero temperature, Phys. Rev. D 72 (2005) 054510 [hep-lat/0409016] [INSPIRE].

[20] F. Farchioni et al., Twisted mass quarks and the phase structure of lattice QCD, Eur. Phys. J. C 39 (2005) 421 [hep-lat/0406039] [INSPIRE].

[21] F. Farchioni et al., The Phase structure of lattice QCD with Wilson quarks and renormalization group improved gluons, Eur. Phys. J. C 42 (2005) 73 [hep-lat/0410031] [INSPIRE].

[22] F. Farchioni et al., Lattice spacing dependence of the first order phase transition for dynamical twisted mass fermions, Phys. Lett. B 624 (2005) 324 [hep-lat/0506025] [INSPIRE].

[23] F. Farchioni et al., Numerical simulations with two flavors of twisted-mass Wilson quarks and DBW2 gauge action, Eur. Phys. J. C 47 (2006) 453 [hep-lat/0512017] [INSPIRE].

[24] T. Chiarappa et al., Numerical simulation of QCD with $u, d, s$ and $c$ quarks in the twisted-mass Wilson formulation, Eur. Phys. J. C 50 (2007) 373 [hep-lat/0606011] [INSPIRE].

[25] A. Deuzeman, U. Wenger and J. Wuilloud, Spectral properties of the Wilson Dirac operator in the $\epsilon$-regime, JHEP 12 (2011) 109 [arXiv:1110.4002] [INSPIRE].

[26] P. Damgaard, U. Heller and K. Splittorff, Finite-Volume Scaling of the Wilson-Dirac Operator Spectrum, Phys. Rev. D 85 (2012) 014505 [arXiv:1110.2851] [INSPIRE]. 
[27] P. Damgaard, U. Heller and K. Splittorff, New Ways to Determine Low-Energy Constants with Wilson Fermions, Phys. Rev. D 86 (2012) 094502 [arXiv: 1206.4786] [InSPIRE].

[28] P.H. Damgaard, U.M. Heller and K. Splittorff, Wilson chiral perturbation theory, Wilson-Dirac operator eigenvalues and clover improvement, arXiv:1301.3099 [INSPIRE].

[29] P. Damgaard, K. Splittorff and J. Verbaarschot, Microscopic Spectrum of the Wilson Dirac Operator, Phys. Rev. Lett. 105 (2010) 162002 [arXiv:1001.2937] [INSPIRE].

[30] G. Akemann, P. Damgaard, K. Splittorff and J. Verbaarschot, Spectrum of the Wilson Dirac Operator at Finite Lattice Spacings, Phys. Rev. D 83 (2011) 085014 [arXiv:1012.0752] [INSPIRE].

[31] K. Splittorff and J. Verbaarschot, The Wilson Dirac Spectrum for QCD with Dynamical Quarks, Phys. Rev. D 84 (2011) 065031 [arXiv:1105.6229] [InSPIRE].

[32] K. Splittorff and J. Verbaarschot, Progress on the Microscopic Spectrum of the Dirac Operator for QCD with Wilson Fermions, PoS (LATTICE 2011) 113 [arXiv:1112.0377] [INSPIRE].

[33] G. Akemann and T. Nagao, Random Matrix Theory for the Hermitian Wilson Dirac Operator and the chGUE-GUE Transition, JHEP 10 (2011) 060 [arXiv:1108.3035] [INSPIRE].

[34] K. Splittorff and J. Verbaarschot, The Microscopic Twisted Mass Dirac Spectrum, Phys. Rev. D 85 (2012) 105008 [arXiv:1201.1361] [INSPIRE].

[35] M. Kieburg, K. Splittorff and J. Verbaarschot, The Realization of the Sharpe-Singleton Scenario, Phys. Rev. D 85 (2012) 094011 [arXiv:1202.0620] [INSPIRE].

[36] G. Akemann and A. Ipsen, Individual Eigenvalue Distributions for the Wilson Dirac Operator, JHEP 04 (2012) 102 [arXiv:1202.1241] [INSPIRE].

[37] K. Splittorff, Chiral Dynamics With Wilson Fermions, PoS (LATTICE 2012)018 [arXiv: 1211.1803] [INSPIRE].

[38] S. Necco and A. Shindler, Spectral density of the Hermitean Wilson Dirac operator: a NLO computation in chiral perturbation theory, JHEP 04 (2011) 031 [arXiv:1101.1778] [INSPIRE].

[39] S. Necco and A. Shindler, On the spectral density of the Wilson operator, PoS (LATTICE 2011) 250 [arXiv: 1108.1950] [INSPIRE].

[40] S. Necco and A. Shindler, Corrections to the Banks-Casher relation with Wilson quarks, arXiv: 1302.5595 [INSPIRE].

[41] S. Aoki, O. Bär and B. Biedermann, Pion scattering in Wilson chiral perturbation theory, Phys. Rev. D 78 (2008) 114501 [arXiv:0806.4863] [INSPIRE].

[42] F. Bernardoni, J. Bulava and R. Sommer, Determination of the Wilson ChPT low energy constant $c_{2}$, PoS (LATTICE 2011) 095 [arXiv:1111.4351] [INSPIRE].

[43] M.T. Hansen and S.R. Sharpe, Determining low-energy constants in partially quenched Wilson chiral perturbation theory, Phys. Rev. D 85 (2012) 054504 [arXiv:1112.3998] [INSPIRE].

[44] O. Bär, G. Rupak and N. Shoresh, Simulations with different lattice Dirac operators for valence and sea quarks, Phys. Rev. D 67 (2003) 114505 [hep-lat/0210050] [INSPIRE].

[45] O. Bär, G. Rupak and N. Shoresh, Chiral perturbation theory at $O\left(a^{2}\right)$ for lattice $Q C D$, Phys. Rev. D 70 (2004) 034508 [hep-lat/0306021] [INSPIRE]. 
[46] K. Cichy, V. Drach, E. Garcia-Ramos, G. Herdoiza and K. Jansen, Overlap valence quarks on a twisted mass sea: a case study for mixed action Lattice QCD, Nucl. Phys. B 869 (2013) 131 [INSPIRE].

[47] M.T. Hansen and S.R. Sharpe, Constraint on the Low Energy Constants of Wilson Chiral Perturbation Theory, Phys. Rev. D 85 (2012) 014503 [arXiv:1111.2404] [INSPIRE].

[48] M. Golterman, Applications of chiral perturbation theory to lattice QCD, arXiv:0912.4042 [INSPIRE].

[49] G. Münster, On the phase structure of twisted mass lattice QCD, JHEP 09 (2004) 035 [hep-lat/0407006] [INSPIRE].

[50] L. Scorzato, Pion mass splitting and phase structure in twisted mass QCD, Eur. Phys. J. C 37 (2004) 445 [hep-lat/0407023] [inSPIRE].

[51] S.R. Sharpe and J.M. Wu, The phase diagram of twisted mass lattice QCD, Phys. Rev. D 70 (2004) 094029 [hep-lat/0407025] [INSPIRE].

[52] G. Colangelo, U. Wenger and J.M. Wu, Twisted mass finite volume effects, Phys. Rev. D 82 (2010) 034502 [arXiv: 1003.0847] [INSPIRE].

[53] O. Bär, Chiral logs in twisted mass lattice QCD with large isospin breaking, Phys. Rev. D 82 (2010) 094505 [arXiv: 1008.0784] [INSPIRE].

[54] S. Ueda and S. Aoki, Chiral perturbation theory for twisted mass QCD at small quark mass, arXiv: 1109.0073 [INSPIRE].

[55] G. Münster and T. Sudmann, Twisted mass chiral perturbation theory for $2+1+1$ quark flavours, JHEP 04 (2011) 116 [arXiv:1103.1494] [INSPIRE].

[56] Alpha collaboration, R. Frezzotti, P.A. Grassi, S. Sint and P. Weisz, Lattice QCD with a chirally twisted mass term, JHEP 08 (2001) 058 [hep-lat/0101001] [INSPIRE].

[57] R. Frezzotti and G. Rossi, Chirally improving Wilson fermions. 1. O(a) improvement, JHEP 08 (2004) 007 [hep-lat/0306014] [INSPIRE].

[58] XLF collaboration, K. Jansen, A. Shindler, C. Urbach and I. Wetzorke, Scaling test for Wilson twisted mass QCD, Phys. Lett. B 586 (2004) 432 [hep-lat/0312013] [INSPIRE].

[59] XLF collaboration, K. Jansen, M. Papinutto, A. Shindler, C. Urbach and I. Wetzorke, Light quarks with twisted mass fermions, Phys. Lett. B 619 (2005) 184 [hep-lat/0503031] [INSPIRE].

[60] XLF collaboration, K. Jansen, M. Papinutto, A. Shindler, C. Urbach and I. Wetzorke, Quenched scaling of Wilson twisted mass fermions, JHEP 09 (2005) 071 [hep-lat/0507010] [INSPIRE].

[61] A.M. Abdel-Rehim, R. Lewis and R. Woloshyn, Spectrum of quenched twisted mass lattice QCD at maximal twist, Phys. Rev. D 71 (2005) 094505 [hep-lat/0503007] [INSPIRE].

[62] ETM collaboration, C. Urbach, Lattice QCD with two light Wilson quarks and maximally twisted mass, PoS (LATTICE 2007) 022 [arXiv:0710.1517] [INSPIRE].

[63] ETM collaboration, P. Dimopoulos, R. Frezzotti, G. Herdoiza, C. Urbach and U. Wenger, Scaling and low energy constants in lattice $Q C D$ with $N_{f}=2$ maximally twisted Wilson quarks, PoS(LATTICE 2007) 102 [arXiv:0710.2498] [INSPIRE].

[64] ETM collaboration, C. Alexandrou et al., Light baryon masses with dynamical twisted mass fermions, Phys. Rev. D 78 (2008) 014509 [arXiv:0803.3190] [InSPIRE]. 
[65] ETM collaboration, R. Baron et al., Light Meson Physics from Maximally Twisted Mass Lattice QCD, JHEP 08 (2010) 097 [arXiv:0911.5061] [INSPIRE].

[66] ETM collaboration, V. Drach, K. Jansen, J. Carbonell, M. Papinutto and C. Alexandrou, Low lying baryon spectrum with $N_{f}=2+1+1$ dynamical twisted quarks, PoS (LATTICE 2010) 101 [arXiv: 1012.3861] [INSPIRE].

[67] G. Herdoiza, Towards Four-Flavour Dynamical Simulations, PoS(LATTICE 2010) 010 [arXiv: 1103.1523] [INSPIRE].

[68] XLF collaboration, K. Jansen et al., Flavor breaking effects of Wilson twisted mass fermions, Phys. Lett. B 624 (2005) 334 [hep-lat/0507032] [INSPIRE].

[69] R. Frezzotti and G. Rossi, $O\left(a^{2}\right)$ cutoff effects in Wilson fermion simulations, PoS (LAT 2007) 277 [arXiv: 0710.2492] [INSPIRE].

[70] ETM collaboration, P. Dimopoulos, R. Frezzotti, C. Michael, G. Rossi and C. Urbach, $O\left(a^{2}\right)$ cutoff effects in lattice Wilson fermion simulations, Phys. Rev. D 81 (2010) 034509 [arXiv:0908.0451] [INSPIRE].

[71] ETM collaboration, C. Michael and C. Urbach, Neutral mesons and disconnected diagrams in Twisted Mass QCD, PoS(LAT 2007)122 [arXiv:0709.4564] [INSPIRE].

[72] ETM collaboration, P. Boucaud et al., Dynamical Twisted Mass Fermions with Light Quarks: Simulation and Analysis Details, Comput. Phys. Commun. 179 (2008) 695 [arXiv: 0803.0224] [INSPIRE].

[73] R. Frezzotti and G. Rossi, Chirally improving Wilson fermions. II. Four-quark operators, JHEP 10 (2004) 070 [hep-lat/0407002] [INSPIRE].

[74] R. Frezzotti and G. Rossi, Twisted mass lattice QCD with mass nondegenerate quarks, Nucl. Phys. Proc. Suppl. 128 (2004) 193 [hep-lat/0311008] [INSPIRE].

[75] K. Osterwalder and E. Seiler, Gauge Field Theories on the Lattice, Annals Phys. 110 (1978) 440 [INSPIRE].

[76] C. Morningstar and M.J. Peardon, Analytic smearing of SU(3) link variables in lattice QCD, Phys. Rev. D 69 (2004) 054501 [hep-lat/0311018] [InSPIRE].

[77] B. Sheikholeslami and R. Wohlert, Improved continuum limit lattice action for QCD with Wilson fermions, Nucl. Phys. B 259 (1985) 572 [INSPIRE].

[78] P. Weisz, Continuum limit improved lattice action for pure Yang-Mills theory. 1., Nucl. Phys. B 212 (1983) 1 [INSPIRE].

[79] P. Weisz and R. Wohlert, Continuum limit improved lattice action for pure Yang-Mills theory. 2., Nucl. Phys. B 236 (1984) 397 [Erratum ibid. B 247 (1984) 544] [InSPIRE].

[80] Y. Iwasaki, Renormalization group analysis of lattice theories and improved lattice action: two-dimensional nonlinear $O(N) \sigma$-model, Nucl. Phys. B 258 (1985) 141 [INSPIRE].

[81] Y. Iwasaki, K. Kanaya, T. Kaneko and T. Yoshie, Scaling in SU(3) pure gauge theory with a renormalization group improved action, Phys. Rev. D 56 (1997) 151 [hep-lat/9610023] [INSPIRE].

[82] Y. Iwasaki, Renormalization Group Analysis of Lattice Theories and Improved Lattice Action. II. Four-dimensional non-Abelian SU(N) gauge model, arXiv:1111.7054 [INSPIRE].

[83] ETM collaboration, R. Baron et al., Light hadrons from $N_{f}=2+1+1$ dynamical twisted mass fermions, PoS (LATTICE 2010) 123 [arXiv:1101.0518] [INSPIRE]. 
[84] ETM collaboration, M. Constantinou et al., $B_{K}$-parameter from $N_{f}=2$ twisted mass lattice QCD, Phys. Rev. D 83 (2011) 014505 [arXiv:1009.5606] [InSPIRE].

[85] ETM collaboration, F. Farchioni et al., Pseudoscalar decay constants from $N_{f}=2+1+1$ twisted mass lattice QCD, PoS(LATTICE 2010) 128 [INSPIRE].

[86] ETM collaboration, B. Blossier et al., Average up/down, strange and charm quark masses with $N_{f}=2$ twisted mass lattice QCD, Phys. Rev. D 82 (2010) 114513 [arXiv:1010.3659] [INSPIRE].

[87] K. Cichy, G. Herdoiza and K. Jansen, Continuum Limit of Overlap Valence Quarks on a Twisted Mass Sea, Nucl. Phys. B 847 (2011) 179 [arXiv:1012.4412] [InSPIRE].

[88] P. Dimopoulos, H. Simma and A. Vladikas, Quenched $B_{K}$-parameter from Osterwalder-Seiler tmQCD quarks and mass-splitting discretization effects, JHEP 07 (2009) 007 [arXiv: 0902 .1074] [INSPIRE]. 